\title{
Geoelectrical Imaging for Shallow Site Investigation at Ekiugbo in Uhunmwode Local Government Area of Edo State, Nigeria
}

\section{${ }^{* 1}$ AIGBOGUN, CO; ${ }^{1}$ ALILE, OM; ${ }^{2}$ EGBAI, JC}

\author{
${ }^{1}$ University of Benin, Department of Physics, Benin City \\ ${ }^{2}$ Delta State University, Department of Physics, Abraka \\ Corresponding Authors: caigbogun@uniben.edu \\ mondayalile@uniben.edu,jamesegbai@yahoo.com
}

\begin{abstract}
A geoelectrical imaging survey was conducted at Ekiugbo in Uhunmwode Local Government Area of Edo State, Nigeria, for Shallow site investigation in order to determine the subsurface lithology using Pole-Dipole array and SAS 1000 Terrameter for the acquisition of field data. Global position system (GPS) was used to geolocate the position of the data acquisition as longitude $005^{\circ} 06^{\prime} 20.6^{\prime \prime}$ latitude $05^{0} 39^{\prime} 24.7^{\prime \prime}$. The data were subjected to an inversion using RES2DINV computer software for analysis and interpretation. The lateral spread was $280 \mathrm{~m}$ and the vertical penetration was $184 \mathrm{~m}$. The resistivities of the various lithological profiles ranges $138 \Omega \mathrm{m}-54938 \Omega \mathrm{m}$. C JASEM https://dx.doi.org/10.4314/jasem.v21i5.12
\end{abstract}

Key words: Geoelectric, GPS, heterogeneous. imaging, lithology, res2DINV, resistivity, subsurface,

The knowledge of subsurface geology and the characterization of the spatial distribution of subsurface physical properties are important for effective delineation of an area under survey (Ahzegbobor, 2010 ) and (Aigbogun, 2010).

The choice of a particular geophysical method depends mainly on the objective of the investigation relative to the sensitivity of the method, the resolution desired, the site conditions, time required for the survey, and the funds and computational resources available. Different geophysical techniques as well as available hydrogeological and geological data are often integrated to obtain a better understanding of the subsurface media at different scales and resolutions. (Meju, 2000) and (Pedersen et al., 2005).

The study of weathering profile, its vertical variation, spatial distribution, textural characteristics of the constituent materials are essential step towards a better understanding of shallow site investigation in complex areas. Due to the heterogeneous nature of the subsurface, the geology of the subsurface needs to be investigated in considerable details (Le Grand, 1962; Asseez, 1972).

This knowledge has led to the application of geophysical methods mostly Electrical resistivity imaging in site investigation. Due to the limitations of the conventional 1-D resistivity sounding and profiling, electrical resistivity imaging (2-D) was used in this study for mapping the subsurface layers at Ekiugbo (Uhunmwode Local Government Area) in Edo State, Nigeria because it is capable of yielding adequate information on subsurface rock types and distribution.
Geology of the study area The study area Ekiugbo village is in Uhunmwode local government area of Edo State which lies north of Benin City within longitude $5^{\circ} 45^{\prime} \mathrm{E}$ and $6^{\circ} 00^{\prime} \mathrm{E}$ and latitudes $6^{\circ} 15^{\prime} \mathrm{N}$ and $6^{\circ} 45^{\circ} \mathrm{N}$. Geologically, the study area is basically sand witched between the Niger-Delta basin and Anambra basin. It lies within the aquiferous Benin formation of the Niger-Delta basin and aquiferous Ogwashi-Asaba formation of the Anambra basin. (Short et al., 1967).

Climate The community lies within the tropical rain forest belt and Rainfall in this area is heavy, varying from an average of $2000 \mathrm{~mm}$ a year. Temperature values ranges between $27^{\circ} \mathrm{C}$ and $34^{\circ} \mathrm{C}$. Edo State has a tropical climate, characterized by two distinct seasons, the wet and dry seasons. The wet season last from April to November and the dry season from December to March. Rainfall intensity decreases from the south to the north. The dry season sets in as a result of the influence of the north-east trade wind.

Theory: In the 2D resistivity method, a large number of measurements are made using different electrode configurations in order to map the subsurface resistivity at different depths below the survey line. We then attempt to determine the true resistivity of the subsurface from the apparent resistivity measurements. One method that is widely used is the linearized least-squares optimization method. (deGroot et al., 1990) and (Ellis et al., 1994), where the relationship between the measured data and model parameters is given by the following equation.

$\left(G^{T}+C\right) \Delta r_{i}=G^{T} \mathrm{~g}-C r_{i-1}$ 
The Jacobian matrix $G$ contains the logarithmic sensitivities of the measurements with respect to the model parameters. The matrix $\mathrm{C}$ contains the damping factors, roughness filters and other constraints, while $\mathrm{g}$ is the data misfit vector containing the difference between the logarithms of the measured and calculated apparent resistivity values. $\boldsymbol{r}_{\boldsymbol{i}-\mathbf{1}}$ is the model parameter vector (the logarithm of the model resistivity values) for the previous iteration, while $\Delta \boldsymbol{r}_{\boldsymbol{i}}$ is change in the model parameters. Using linear approximations, it can be shown an estimate of the model resolution matrix (Menke, 1984) is given by; $\mathbf{R}=\left(\mathbf{G}^{\mathbf{T}} \mathbf{G}+\mathbf{C}\right)^{-1} \mathbf{G}^{\mathbf{T}} \mathbf{G}$

Electrical resistivity tomography (ERT) is one of the oldest and most commonly used geophysical methods and measures the variation of electrical resistivity in the subsurface (Dahlin, 2001). The ERT technique has evolved significantly from its basic form as 1D electrical prospecting method that was used to map vertical or horizontal layers or anomalies in the subsurface (Slichter, 1993). Over the past two decades, coupled with the significant advancements in computers, there have been revolutionary improvements in ERT and it is now one of the most widely researched and applied techniques in geophysics (Revil, et al., 2012) and (Loke, et al., 2013).

The fundamental physical law used in resistivity surveys is Ohm's law that governs the flow of electrical current in the earth. The equation for Ohm's law in vector form for current flow in a continuous medium is given by (Tsourlos, 1995).

$J_{c}=$

where $\sigma$ is the electrical conductivity of the medium, $J_{c}$ is the current density and $E$ is the electric field intensity. In practice, what is measured is the electric field potential $U$. The relationship between the electric potential and the field intensity is given by:

$\nabla x E+\frac{\partial B}{\partial t}=0$

For DC current, $\frac{\partial \mathrm{B}}{\partial \mathrm{t}}=0$ and

$\mathrm{E}=-\nabla \mathrm{U}$

U must satisfy Laplace's equation and $U$ is said to be harmonic

$-\nabla^{2} \mathrm{U}=0$

Combining Equations 1 and 2 we get:

$J_{c}=-\sigma \nabla U$
In almost all surveys, the current sources are in the form of point sources. In this case, over an elemental volume $\nabla V$ surrounding a current source $I_{c}$ located at $\left(x_{s}, y_{s}, z_{s}\right)$, the relationship between the current density and the current is given by Dey, et al., (1979):

$\nabla J_{c}=\left(\frac{1}{\nabla V}\right) \delta\left(x-x_{s}\right)\left(y-y_{s}\right)\left(z-z_{s}\right)$ 4

where $\delta$ is the Dirac delta function. Equation 4 can then be rewritten as:

$$
\begin{aligned}
& -\nabla \cdot[\sigma(x, y, z) \nabla U(x, y, z)]=\left[\frac{1}{\nabla V}\right] \delta\left(x-x_{s}\right)(y- \\
& \left.y_{s}\right)\left(z-z_{s}\right)
\end{aligned}
$$

This partial differential equation gives the subsurface potential distribution in an isotropic, non-uniform 3D medium due to a point current source. Numerous techniques have been developed to solve this equation. This is the 'forward' modeling problem which determines the potential that would be observed over a given subsurface structure, and is incorporated in both forward models and inversion algorithms.

The electrical potential depends on distance, not direction, so it is appropriate to write Laplace's equation in spherical coordinates with an rdependence only.

$\frac{1}{\mathrm{r}^{2}}\left[\frac{\partial}{\partial \mathrm{r}}\left(\mathrm{r}^{2} \frac{\partial \mathrm{u}}{\partial \mathrm{r}}\right)\right]=0$

This has a solution; $U=\frac{A}{r}+B$

where $\mathrm{A}$ and $\mathrm{B}$ are constants.

Since the potential must go to zero a great distance from the current source, it is obvious that $\mathrm{B}=0$.

Solution for a point current source in uniform unbounded space

$U=\frac{I \rho}{4 \pi r}$

Two dimensional electrical imaging surveys model are more accurate than $1 \mathrm{D}$ resistivity sounding of surveys as it allows horizontal as well as vertical resistivity variations. Loke, (2004).

\section{MATERIAL AND METHODS}

The Pole-Dipole array and SAS 1000 Terrameter was used for the acquisition of field data. Global position system (GPS) was used to geolocate the position of the data acquisition as longitude $005^{\circ} 06^{\prime 2} 20.6^{\prime \prime}$ latitude 
$05^{0} 39$ '24.7'. The data were subjected to an inversion using RES2DINV computer software for interpretation and analysis. Figure 1, represent the pole-dipole field work configuration. The $\mathrm{C} 2$ is a remote electrode that was placed sufficiently far from the survey line. The effect of the $\mathrm{C} 2$ electrode proportionality to the square of the ratio of C1-P1 distance to the $\mathrm{C} 2-\mathrm{P} 1$ distance was maintained during field work. The lateral spread was $280 \mathrm{~m}$.

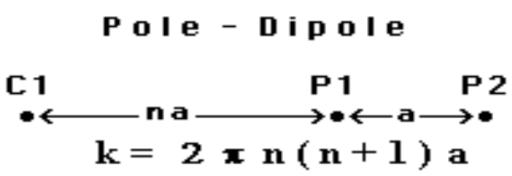

Fig 1: Pole-dipole array

\section{RESULTS AND DISCUSSION}

The result of the interpretation is shown in Figure 2. The analysis of the interpretation as it is in Figure 2, horizontal stratification of the subsurface earth as it is assumed cannot be substantiated. The essence or importance of a 2-D resistivity tomography is to be able to delineate the extent of the subsurface materials horizontally and vertically in an inhomogeneous earth. The resistivity ranges from $138 \Omega \mathrm{m}-54938 \Omega \mathrm{m}$, the lateral spread and vertical depth of penetration are $280 \mathrm{~m}$ and $183.6 \mathrm{~m}$ respectively. Looking at the model image, there are features at the subsurface that their resistivity falls in between the range which signifies the presence of earth materials in the environment of study. There is an oval structure that has the shape of a lobe of different dimensions spreading laterally from $50 \mathrm{~m}-$ $180 \mathrm{~m}$ and thickness of $33.7 \mathrm{~m}$, with a very high resistivity values of $23154 \Omega \mathrm{m}$ and $54938 \Omega \mathrm{m}$ respectively.

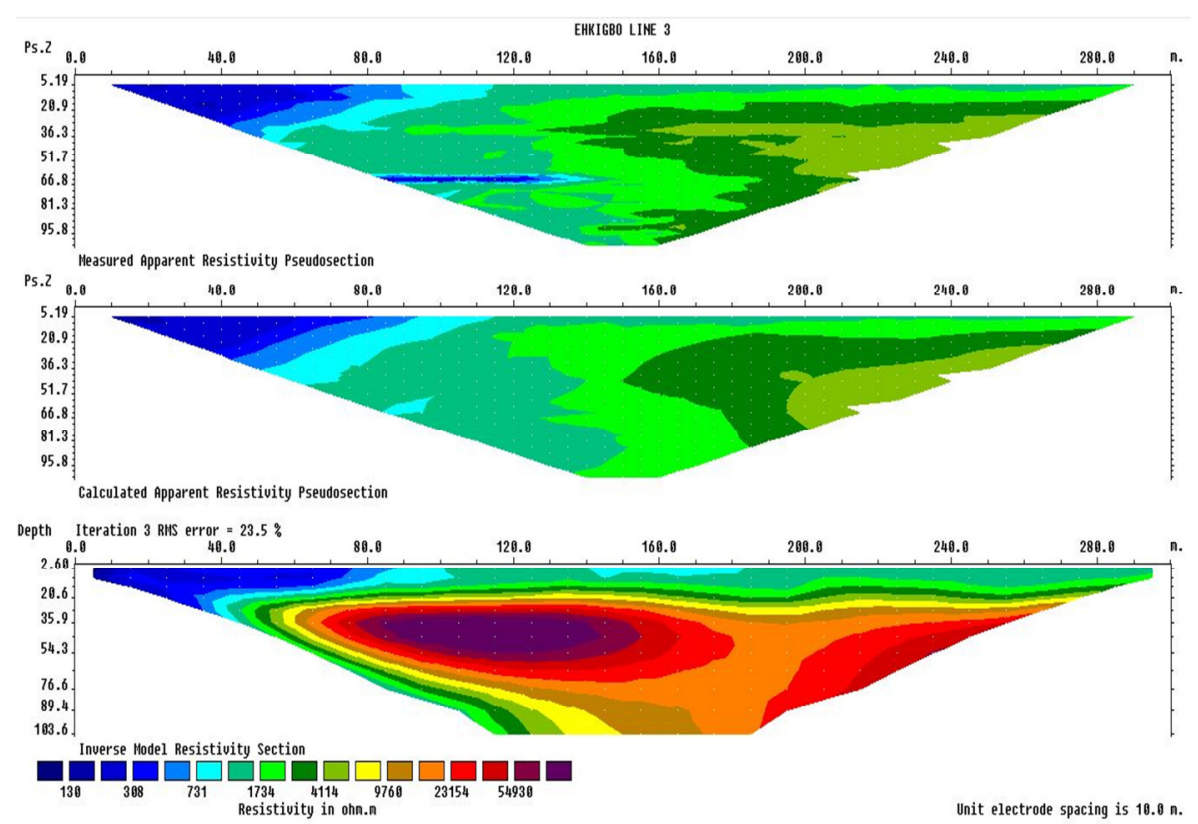

Fig 2:Resistivity model Image of the Surveyed Area

Conclusion The resistivities of the various lithological profiles ranges $138 \Omega \mathrm{m}-54938 \Omega \mathrm{m}$. There is an oval structure identified that has the shape of a lobe of different dimensions spreading laterally from $50 \mathrm{~m}-180 \mathrm{~m}$ and thickness of $33.7 \mathrm{~m}$, with a very high resistivity values of $23154 \Omega \mathrm{m}$ and 54938 $\Omega \mathrm{m}$ respectively. This indicates the presence of mineral deposit which require further investigations.

\section{REFERENCES}

Aigbogun, CO (2010). Geoelectric Investigation of the Groundwater Potential in Uhunmwode Local Government Area, Edo State, Nigeria. Ph.D Thesis, Department of Physics, University of Benin, 255pp.

Ahzegbobor, PA (2010). Acquisition geometry and inversion of 3-d geoelectrical resistivity imaging data for environmental and engineering 
investigations. Ph.D thesis, Covenant University, Ota.

Asseez, LO (1972). Rural water supply in the basement complex of Western State, Nigeria. Hydrogeological Sciences Bulletin, Des Sciences Hydrologiques XVII, 14: 97-110.

Dahlin, T (2001). The development of DC resistivity imaging techniques. Computer and Geosciences, 27(9): 1019-1029.

deGroot-Hedlin and Constable (1990). Occam's inversion to generate smooth, two-dimensional models from magnetotelluric data. Geophysics $55,1613-1624$.

Dey and Morrison (1979). Resistivity modelling for arbitrarily shaped two-dimensional structures. Geophys. Prospect. 27, 106-136.

Ellis and Oldenburg (1994). Applied geophysical inversion. Geophysical Journal International 116, $5-11$.

Loke, MH (2004). Tutorial: 2-D and 3-D electrical imaging surveys. Geotomo Software, Penang, Malaysia.

Loke, MH; Chambers, JE; Rucker, DF; Kuras, O; Wilkinson, PB (2013). Recent developments in the direct-current geoelectrical imaging method. Journal of Applied Geophysics, 95: 135-156.

Meju, MA (2000). Environmental geophysics: tasks ahead. Journal of Applied Geophysics, 44, p. 6365 .

Menke, W (1984). Geophysical data analysis: Discrete Inverse Theory. Academic Press, London.

Pedersen, LB; Bastani, M; Dynesius, L (2005). Groundwater exploration using combined controlled-source and radiomagnetotelluric techniques. Geophysics, 70, p. G8-G15.

Revil, A; Karaoulis, M; Johnson, T; Kemna. A (2012). Review: Some low- frequency electrical methods for subsurface characterization and monitoring in hydrogeology. Hydrogeology Journal, 20(4): 617-658.

Short and Stauble (1967). Outline geology of Niger delta A.A. PG-V.5. I P. $761-799$.

Slichter, LB (1933). The interpretation of the resistivity prospecting method for horizontal structures, J Appl Phys, v4, pp 307-322.

Tsourlos, P (1995). Modeling, Interpretation and Inversion of Multielectrode Resistivity Data. D.Phil. Thesis, University of York, 315 pp. 